\title{
Shall we call them "telomere-mediated"? Renaming the idiopathic after the cause is found
}

\author{
Samantha J. Merck ${ }^{1,2}$ and Mary Armanios ${ }^{1,3}$
}

Affiliations: ${ }^{1}$ Dept of Oncology, Johns Hopkins University School of Medicine, Baltimore, MD, USA. ${ }^{2}$ Division of Pulmonary and Critical Care Medicine, Johns Hopkins University School of Medicine, Baltimore, MD, USA. ${ }^{3}$ McKusick-Nathans Institute of Genetic Medicine, Johns Hopkins University School of Medicine, Baltimore, MD, USA.

Correspondence: Mary Armanios, MD, 1650 Orleans St., CRB 1 Room 186, Baltimore, MD 21287, USA. E-mail: marmani1ajhmi.edu

0 @ERSpublications

This editorial contextualises the findings of two articles in this ERJ issue, and proposes new terminology http://ow.ly/SRcc305Mvbn

Short of lung scarring, the idiopathic interstitial pneumonias (IIPs) primarily share their poorly understood aetiology. Attempts to identify features that refine the distinctions between IIP diagnostic categories, based on pathologic and radiographic criteria, have led to recent revisions in their evaluation [1, 2]. The consensus recommendation relies on multidisciplinary assessment to clarify an IIP diagnosis [2]. However, there still remains a relatively low concordance rate across groups of experienced clinicians $[3,4]$ and the heterogeneity of the clinical course within IIP subtypes poses a challenge to using these categories when making decisions at the bedside [2].

Over the past decade, it has become evident that abnormally short telomeres are a causal risk factor for pulmonary fibrosis in a large subset of patients [5-18]. Mutations in the genes that code for the telomerase enzyme essential components, TERT and TR (also known as TERC), are the most common cause of familial pulmonary fibrosis $[8,10,16]$. Five other telomere-related genes have recently been found to be mutated in families with pulmonary fibrosis: DKC1, TINF2, RTEL1, PARN and NAF1 [6, 13, 14, 17-19]. Mutations in these seven genes explain the inheritance in at least one-third of families. They are also a risk factor for disease in a smaller subset of pulmonary fibrosis patients without a family history $[5,15]$. Patients with telomere-mediated lung disease show extrapulmonary manifestations that follow a syndromic pattern [7]. These short telomere syndrome features most commonly appear in varying degrees of bone marrow failure and liver disease, often complicated by hepatopulmonary syndrome, among other features [11, 20-23]. Within families with autosomal dominant inheritance, genetic anticipation, which is defined by an earlier and more severe onset of disease in successive generations, may be seen. The change in onset across generations depends on the extent of the loss-of-function caused by a mutation, with more deleterious mutations causing obvious genetic anticipation in two or three generations, while hypomorphic mutations show more subtle changes in the same timeframe $[9,24]$. The progressive shortening of telomeres across generations underlies this earlier onset of disease [7]. Recognising this subset of patients is increasingly recognised to have implications for patient care. One of the most important examples is in the lung transplantation setting, where recognising patients with telomere-mediated lung disease is critical because it predicts potentially avoidable complications related to myelosuppression, infection and renal failure [25].

The question of the type of lung disease developed by mutation carriers with short telomeres has been an important one, since this subset comprises the largest genetically homogeneous group of pulmonary fibrosis patients. A review of the literature in 2012 found the idiopathic pulmonary fibrosis/usual

Received: Oct 282016 | Accepted: Oct 282016

Conflict of interest: None declared.

Copyright OERS 2016 
interstitial pneumonia (IPF/UIP) phenotype to be most common in telomerase mutation carriers, accounting for $65-70 \%$ of cases [26]. However, the lung disease spectrum described in these patients is broad. It includes the IIP subtypes as well as phenotypes that are not considered IIPs such as hypersensitivity pneumonitis. Emphysema, which is classically considered to have a distinct aetiology, is also a manifestation of telomere-mediated lung disease and may be a common presentation in mutation carriers who smoke, appearing either alone, or combined with pulmonary fibrosis [19].

In this issue of the European Respiratory Journal, two articles add to the literature in this area $[27,28]$. The paper by NEwTON et al. [27] summarises the clinical characteristics of 115 individuals from 64 families with telomerase and telomere gene mutations. Among them, 77 individuals from 15 families had undergone multidisciplinary assessment, and, within this group, $80 \%$ of families had discordant lung disease diagnoses. The pathology included the previously reported IIPs, hypersensitivity pneumonitis and emphysema. There were also a number of female cases with pleuroparenchymal fibroelastosis, an otherwise rare IIP that is known to have familial clustering [29]. Although the authors focused their study on patients with mutations in four genes (TERT, TR, RTEL1 and PARN), the literature supports this heterogeneity in other short telomere families [30]. In a prior landmark study, STEELE et al. [31] reported discordance in the IIP diagnosis in nearly half of 111 families studied. In their study, Newton et al. further compared the survival time or time to lung transplant in patients with IPF and non-IPF diagnoses and found they were similar with a median around 3 years. These data are in contrast to the idea that the IPF pattern itself predicts worse outcomes. They suggest that, within this homogeneous subset of telomere-mediated lung disease patients, the genetic diagnosis may be more prognostic than multidisciplinary assessment based on radiographic and pathologic information.

In their article, BORIE et al. [28] report on a national cohort from France with 237 centrally evaluated patients with pulmonary fibrosis of suspected genetic origin. This is one of the largest systematic efforts to examine the prevalence of telomerase mutations in this population. Patients with a family history of pulmonary fibrosis as well as sporadic patients with extrapulmonary short telomere syndrome features were included. In this study, only TERT and TR were genotyped, and the $17 \%$ prevalence of rare variants reported is within the documented range seen in similar cohorts $[10,16]$. Within this group, the highest frequency of mutations was in patients who had a personal or family history of short telomere syndrome features including bone marrow failure and liver disease; $42 \%$ of these 38 patients had a rare telomerase gene variant. This finding is consistent with prior evidence that the molecular short telomere defect may, in a subset of patients with lung disease, be clinically recognisable by the presence of extrapulmonary pathology in these patients or their families [11]. It is a reminder of the importance of obtaining a personal and family history for short telomere syndrome features when evaluating pulmonary fibrosis/emphysema patients. As the authors point out, the significance of some disease-associated variants may at times be unclear even when their pathogenicity is stratified based on available evidence, including frequency and prior reports. This challenge exemplifies the hurdle to interpreting genetic information at the bedside, where family-based studies and functional testing for every variant are not available outside of a specialised research setting.

The accompanying studies are reminders of the opportunities and challenges that come with integrating genetic medicine paradigms in clinical practice. Surprisingly, the lung disease phenotypes that appear within families which share a single molecular defect at telomeres span a broad spectrum of disorders we have traditionally considered distinct. This raises the possibility that in some cases we may need to consider a molecular classification that transcends clinical descriptors we have historically utilised. There is already evidence that such an approach may refine patient care algorithms in some cases. For example, in light of the already existing literature in this area, the findings in the Newton study would suggest that there may be settings where a genetic evaluation could precede or even replace a lung biopsy for some patients. If implemented, this could be valuable in not only providing more predictive diagnostic/ prognostic information but also in improving patient outcomes by averting the risk of respiratory complications following surgical procedures as is well-documented for IPF [32, 33]. Considering an umbrella category of "telomere-mediated lung disorders" can also facilitate averting the exquisite sensitivity to commonly used medications and procedures [25,34]. As with the implementation of genetic paradigms in other areas of medicine, there are undoubtedly hurdles. A robust infrastructure for genetic counselling, patient education and the most rigorous algorithms for interpreting genetic variation is not yet widely accessible. Telomere length measurement by the clinically validated flow cytometry and fluorescence in situ hybridisation method is only now becoming increasingly available. Nevertheless, in looking ahead, it seems inevitable that we will one day find it necessary to call these disorders by their real name.

\section{References}

1 American Thoracic Society/European Respiratory Society International Multidisciplinary Consensus Classification of the Idiopathic Interstitial Pneumonias. Am J Respir Crit Care Med 2002; 165: 277-304. 
Travis WD, Costabel U, Hansell DM, et al. An official American Thoracic Society/European Respiratory Society statement: update of the international multidisciplinary classification of the idiopathic interstitial pneumonias. Am J Respir Crit Care Med 2013; 188: 733-748.

3 Flaherty KR, Andrei AC, King TE Jr, et al. Idiopathic interstitial pneumonia: do community and academic physicians agree on diagnosis? Am J Respir Crit Care Med 2007; 175: 1054-1060.

4 Walsh SL, Calandriello L, Sverzellati N, et al. Interobserver agreement for the ATS/ERS/JRS/ALAT criteria for a UIP pattern on CT. Thorax 2016; 71: 45-51.

5 Alder JK, Chen JJ, Lancaster L, et al. Short telomeres are a risk factor for idiopathic pulmonary fibrosis. Proc Natl Acad Sci U S A 2008; 105: 13051-13056.

6 Alder JK, Parry EM, Yegnasubramanian S, et al. Telomere phenotypes in females with heterozygous mutations in the dyskeratosis congenita 1 (DKC1) gene. Hum Mutat 2013; 34: 1481-1485.

7 Armanios M. Syndromes of telomere shortening. Annu Rev Genomics Hum Genet 2009; 10: 45-61.

8 Armanios M. Telomerase and idiopathic pulmonary fibrosis. Mutat Res 2012; 730: 52-58.

9 Armanios M, Chen JL, Chang YP, et al. Haploinsufficiency of telomerase reverse transcriptase leads to anticipation in autosomal dominant dyskeratosis congenita. Proc Natl Acad Sci U S A 2005; 102: 15960-15964.

10 Armanios MY, Chen JJ, Cogan JD, et al. Telomerase mutations in families with idiopathic pulmonary fibrosis. N Engl J Med 2007; 356: 1317-1326.

11 Parry EM, Alder JK, Qi X, et al. Syndrome complex of bone marrow failure and pulmonary fibrosis predicts germline defects in telomerase. Blood 2011; 117: 5607-5611.

12 Stanley SE, Gable DL, Wagner CL, et al. Loss-of-function mutations in the RNA biogenesis factor NAF1 predispose to pulmonary fibrosis-emphysema. Sci Transl Med 2016; 8: 351 ra107.

13 Cogan JD, Kropski JA, Zhao M, et al. Rare variants in RTEL1 are associated with familial interstitial pneumonia. Am J Respir Crit Care Med 2015; 191: 646-5611.

14 Stuart BD, Choi J, Zaidi S, et al. Exome sequencing links mutations in PARN and RTEL1 with familial pulmonary fibrosis and telomere shortening. Nat Genet 2015; 47: 512-517.

15 Cronkhite JT, Xing C, Raghu G, et al. Telomere shortening in familial and sporadic pulmonary fibrosis. Am J Respir Crit Care Med 2008; 178: 729-737.

16 Tsakiri KD, Cronkhite JT, Kuan PJ, et al. Adult-onset pulmonary fibrosis caused by mutations in telomerase. Proc Natl Acad Sci U S A 2007; 104: 7552-7557.

17 Alder JK, Stanley SE, Wagner CL, et al. Exome sequencing identifies mutant TINF2 in a family with pulmonary fibrosis. Chest 2015; 147: 1361-1368.

18 Kropski JA, Mitchell DB, Markin C, et al. A novel dyskerin (DKC1) mutation is associated with familial interstitial pneumonia. Chest 2014; 146: e1-e7.

19 Stanley SE, Chen JJ, Podlevsky JD, et al. Telomerase mutations in smokers with severe emphysema. J Clin Invest 2015; 125: 563-570.

20 Gorgy AI, Jonassaint NL, Stanley SE, et al. Hepatopulmonary syndrome is a frequent cause of dyspnea in the short telomere disorders. Chest 2015; 148: 1019-1026.

21 Jonassaint NL, Guo N, Califano JA, et al. The gastrointestinal manifestations of telomere-mediated disease. Aging cell 2013; 12: 319-323.

22 Stanley SE, Armanios M. The short and long telomere syndromes: paired paradigms for molecular medicine. Curr Opin Genet Dev 2015; 33: 1-9.

23 Calado RT, Regal JA, Kleiner DE, et al. A spectrum of severe familial liver disorders associate with telomerase mutations. PLoS One 2009; 4: e7926.

24 Alder JK, Cogan JD, Brown AF, et al. Ancestral mutation in telomerase causes defects in repeat addition processivity and manifests as familial pulmonary fibrosis. PLoS Genet 2011; 7: e1001352.

25 Silhan LL, Shah PD, Chambers DC, et al. Lung transplantation in telomerase mutation carriers with pulmonary fibrosis. Eur Respir J 2014; 44: 178-187.

26 Armanios M. Telomerase mutations and the pulmonary fibrosis-bone marrow failure syndrome complex. $N$ Engl $J$ Med 2012; 367: 384.

27 Newton CA, Batra K, Torrealba J, et al. Telomere-related lung fibrosis is diagnostically heterogeneous but uniformly progressive. Eur Respir J 2016; 48: 1710-1720.

28 Borie R, Tabèze L, Thabut G, et al. Prevalence and characteristics of TERT and TERC mutations in suspected genetic pulmonary fibrosis. Eur Respir J 2016; 48: 1721-1731.

29 Watanabe K. Pleuroparenchymal fibroelastosis: its clinical characteristics. Curr Respir Med Rev 2013; 9: 299-237.

30 Parry EM, Alder JK, Lee SS, et al. Decreased dyskerin levels as a mechanism of telomere shortening in X-linked dyskeratosis congenita. J Med Genet 2011; 48: 327-333.

31 Steele MP, Speer MC, Loyd JE, et al. Clinical and pathologic features of familial interstitial pneumonia. Am J Respir Crit Care Med 2005; 172: 1146-1152.

32 Ghatol A, Ruhl AP, Danoff SK. Exacerbations in idiopathic pulmonary fibrosis triggered by pulmonary and nonpulmonary surgery: a case series and comprehensive review of the literature. Lung 2012; 190: 373-380.

33 Sakamoto S, Homma S, Mun M, et al. Acute exacerbation of idiopathic interstitial pneumonia following lung surgery in 3 of 68 consecutive patients: a retrospective study. Intern Med 2011; 50: 77-85.

34 Stanley SE, Rao AD, Gable DL, et al. Radiation sensitivity and radiation necrosis in the short telomere syndromes. Int J Radiat Oncol Biol Phys 2015; 93: 1115-1117. 